Journal of Marine Science and Technology; Vol. 17, No. 3; 2017: 271-278

DOI: $10.15625 / 1859-3097 / 17 / 3 / 9803$

http://www.vjs.ac.vn/index.php/jmst

\title{
APPLICATION OF AUTOMATED CALIBRATION METHOD TO CALIBRATE PARAMETERS IN SWAN MODEL USING WAVE HEIGHT DATA FROM SATELLITE AND MSP1 IN EASTERN VIETNAM SEA
}

\author{
Nguyen Trung Thanh ${ }^{1 *}$, Nguyen Minh Huan², Tran Quang Tien ${ }^{3}$ \\ ${ }^{1}$ Northern Centre for Integrated Coastal Management and Planning, \\ Vietnam Administration of Seas and Islands \\ ${ }^{2}$ Hanoi University of Science, $V N U$ \\ ${ }^{3}$ The National Centre for Hydro-Meteorological Forecasting \\ E-mail: thanhnt2212@gmail.com
}

Received: 8-5-2017

\begin{abstract}
Waves are one of the driving forces that have strong and direct impacts on human activities in the oceans, seas and coastal areas. Eastern Vietnam Sea as well as the Vietnamese coastal zone is influenced by the monsoon and storm, so the wave field is also particularly strong and severe in these weather patterns. This article aims to present and apply the automated calibration method to SWAN model with satellite data and real-time data at the oil platform MSP1 of VietSo-Petro Company to find the most suitable parameter set for Eastern Vietnam Sea and to increase the accuracy of wave forecasting in Vietnam. Operations are executed by changing the value of each parameter in the SWAN model and the results are evaluated through the GoF function.
\end{abstract}

Keywords: SWAN, calibration, Eastern Vietnam Sea.

\section{INTRODUCTION}

The research on SWAN model has been carried out in many agencies, research institutes and universities... [1, 2], however, the calibration is usually only made at a fixed station and the calibration parameter mainly focuses on the coefficient for determining the rate of whitecapping dissipation of Komen (CDS2), and obtaining a different parameter with each calibration problem. Therefore this article proceeds to calibrate the parameters of model through the automated calibration method SWAN-CI in OpenDA software to optimize and bring high efficiency in the calibration processes. In addition, the article uses both wave height data from satellite and the oil platform MSP1 of VietSo-Petro Company to complete both space and time calibration to obtain the most appropriate parameter set for Eastern Vietnam Sea.

\section{METHODS OF RESEARCH}

\section{Brief theory of SWAN}

The SWAN wave model is a thirdgeneration spectral wave model, designed to obtain realistic wave estimates for any region of wind generated surface gravity waves. The physics is governed by the wave action equation, Booij et al., [3]:

$$
\frac{\partial N}{\partial t}+\nabla_{\vec{x}}\left[\left(\vec{c}_{g}+\vec{U}\right) N\right]+\frac{\partial c_{\sigma} N}{\partial \sigma}+\frac{\partial c_{\theta} N}{\partial \theta}=\frac{S_{t o t}}{\sigma}(1)
$$

Where: $N(\vec{x}, t, \sigma, \theta)$ is the wave action density spectrum, as a function of the physical space and time $\vec{x}$ and $t, \sigma$ and $\theta$ are the relative 
frequency and the wave direction. The first two terms on the left-hand side of balance equation are the local and advective rates of change of wave action density, where $\vec{c}_{g}$ is the wave group velocity, and $\vec{U}$ is the ambient current velocity. The third term, $\frac{\partial c_{\sigma} N}{\partial \sigma}$ represents the relative frequency shifting due to variations in depth and ambient current. The fourth term, $\frac{\partial c_{\theta} N}{\partial \theta}$ represents the depth-induced and current-induced refraction, where $c_{\sigma}$ and $c_{\theta}$ are the propagation speeds in spectral space $\sigma$ and $\theta$. On the right-hand side, the term $S_{\text {tot }}$ includes all of the source/sink terms and represents all the physical processes that generate, dissipate, and redistribute wave energy, Komen et al., [4].

The source/sink terms are defined via the energy density $E(\sigma, \theta)$. $S_{t o t}$ consists of six main parts:

$$
S_{t o t}=S_{i n}+S_{n l 3}+S_{n l 4}+S_{d s, w}+S_{d s, b}+S_{d s, b r}
$$

Where: $S_{\text {in }}$ denotes the wave growth by the wind; $S_{n l 3}, S_{n l 4}$ represent nonlinear wave energy transfers due to triad and quadruplet wavewave interactions, respectively, $S_{d s, w}, S_{d s, b}, S_{d s, b r}$ are wave energy dissipations due to whitecapping, bottom friction, and depth-induced wave breaking, respectively. Among those terms, the present research will focus on the contribution of wave growth by the wind input $S_{i n}$. It is widely accepted that the transfer of wind energy to the waves may be conceived of as the superposition of resonance, Phillips [5] and positive feedback mechanisms, Miles [6].

The integration of the action balance equation has been implemented in SWAN with both finite difference and finite element schemes in all file dimensions (time, geographic space and spectral space).

\section{Automated calibration method SWAN-CI of Open DA}

OpenDA is an open-source toolbox for developing and applying data assimilation and calibration algorithms to dynamic models [7]. In the calibration or parameter estimation algorithms, the basic idea is to find the set of model parameters which minimizes the cost function measuring the distance between the observation and the model prediction.

Automated calibration method SWAN-CI is part of Open DA [8]. The parameter values that correspond to the minimum value of the GoF (Goodness of Fitting) are considered the optimum parameter values for the given optimisation experiment and the selected optimisation method. For each set of parameter values during parameter variation, the $\mathrm{GoF}$ provides a measure indicating how close the SWAN model results for that set are to observational data.

This study focuses on modifying the SWAN model parameters related to wave height in Eastern Vietnam Sea. Based on general practice and availability of reliable data sets, the GoF will in principle be based on a formulation in terms of the integral parameters of significant wave height:

$$
G o F=\frac{1}{2} \frac{\sum_{i=1}^{N_{i}}\left[\omega_{H_{s}}^{i}\left(H_{s, o b s}^{i}-H_{s, s i m}^{i}\right) / \sigma_{H_{s, b s s}}\right]^{2}}{\sum_{i=1}^{N_{i}} \omega_{H_{s}}^{i}}
$$

In which $H_{s, s i m}^{i}$ and $H_{s, o b s}^{i}$ are significant wave height simulation and observation at $\mathrm{i} ; \omega_{H_{s}}^{i}$ is a weight function (for the present moment taken to be equal to 1$) ; N_{i}$ is the number of spatial calibration data points; $\sigma_{H_{s, o b s}}$ is the standard deviation of the observed parameter in the particular location.

In addition, this study also evaluates the results of the calculation based on the standard deviation of error (STD) according to the following formula:

$$
S T D=\sqrt{\frac{1}{N_{i}} \sum_{i=1}^{N_{i}}\left(\left(H_{s, s i m}^{i}-H_{s, o b s}^{i}\right)-B\right)^{2}}
$$

Where: $B$ is the trend of simulation values, $B$ is calculated by the formula: 


$$
B=\frac{1}{N_{i}} \sum_{i=1}^{N_{i}}\left(H_{s, s i m}^{i}-H_{s, o b s}^{i}\right)
$$

Simulation is performed throughout Eastern
Vietnam Sea with large computational space, so the parameters included in the calibration focus only on parameters that affect deep water waves, as shown in the following table:

Table 1. List of parameters used for calibration

\begin{tabular}{|c|c|c|c|c|c|c|c|}
\hline No & Phys. process & Symbol & Acronym & Default value & Max & Min & Calibration step \\
\hline 1 & & $C_{d s, w c}$ & CDS2 & 0.000236 & 0.0005 & 0.0002 & 0.00002 \\
\hline 2 & Whitecapping & $q$ & powst & 2.0 & 3.0 & 1.0 & 0.2 \\
\hline 3 & & $r$ & powk & 0.0 & 2.0 & 0.0 & 0.2 \\
\hline 4 & Triads & $f_{\max , E B}$ & cutfr & 2.5 & 4.0 & 1.0 & 0.2 \\
\hline
\end{tabular}

\section{Data}

Depth and shoreline data throughout Bien Dong Sea are ETOPO data collected from NOAA [9] and detailed depth data in nearshore areas collected from marine surveys of other study in this area. The domain is the entire Eastern Vietnam Sea stretching from $1.5^{\circ}$ to $25^{\circ} \mathrm{N}$ (from 150,000 to $2,800,000$ in VN2000 coordinate system, projection zone $105^{\text {th }}$ ) and $99^{\circ}$ to $121^{\circ} \mathrm{E}(-150,000$ to $2,200,000$ in VN2000 coordinate system, projection zone $\left.105^{\text {th }}\right)$. The grid is a square grid $25 \times 25 \mathrm{~km}$ with $92 \times 106$ grid cells.

Wind data is the global reanalysis wind CFSv2 of National Center for Environmental Forecasting (NCEP) [10] with a space step of $0.2 \times 0.2$ degrees and a time step of 1 hour from 1979 to present. The wind field was converted to the VN2000 coordinate system and interpolated into gridlines $20 \times 20 \mathrm{~km}$ and time step of 1 hour corresponding to each problem.

The wave data used to calibrate the model parameters were collected from two main sources:

Wave height data were collected from tracks of satellites of the Online Data Extraction Service [11], satellite activity time is shown in the following table 2.

This study uses data from three satellites: Jarson2, HY-2A and Saral-Altica. Each of these satellites has a cycle of about 10 days with a total number of rounds around the earth being about 240 cycles.
Table 2. List of satellites that capture wave heights

\begin{tabular}{|c|c|c|}
\hline No & Satellite name & Activity time \\
\hline 1 & ERS-2 & $1995-2003$ \\
\hline 2 & Geosat - Follow (G2) & $2000-2008$ \\
\hline 3 & Jarson1 & $2002-2013$ \\
\hline 4 & Envisat & $2002-2012$ \\
\hline 5 & Jarson2 & 2008 - now \\
\hline 6 & $\mathrm{HY}-2 \mathrm{~A}$ & 2014 - now \\
\hline 7 & Saral-Altica & 2013 - now \\
\hline 8 & Jarson3 & 2016 - now \\
\hline
\end{tabular}

In order to perform model parameter calibration by satellite wave heights, it is necessary to compile these discrete data for equidistant time steps and for fixed spatial positions. This study uses time step of 1 hour and 174 fixed points. Therefore, the wave height is interpolated as follows:

Select satellites and download track data files of each satellite in the simulation period.

Interpolate wave height data from tracks to 174 points (fig. 1): choose all neighboring points of each point in 174 points with a radius of 0.5 degrees in space and 0.5 hours in time at every hour round to interpolate.

At each point in each time, no data will be assigned a value of -999 so the model does not include these values in the calibration process.

Wave data in the oil platform MSP1 of VietSo-Petro Company, this data were assigned in the $175^{\text {th }}$ point with time step of 1 hour. 
The above data are processed and converted according to input standard of SWAN as well as of OpenDA for calculation.

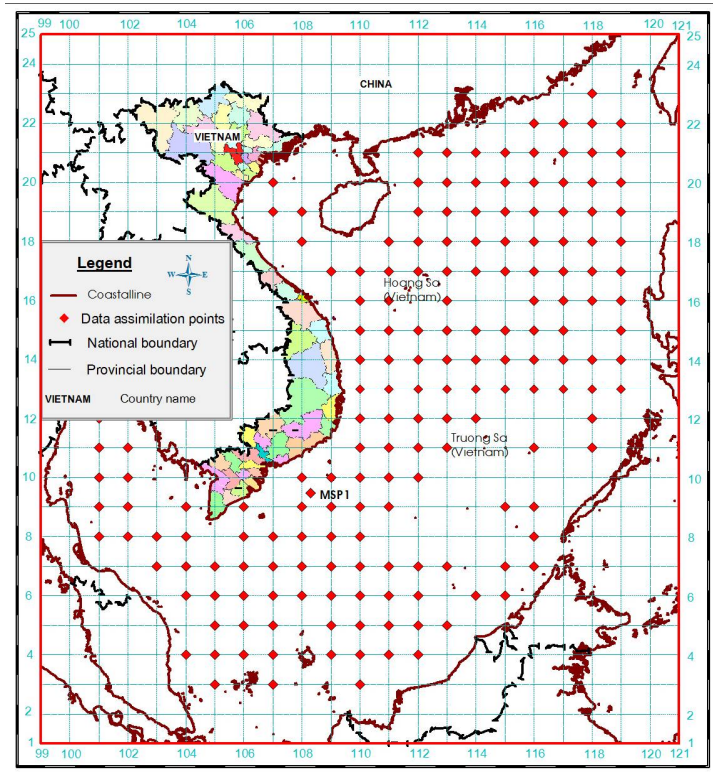

Fig. 1. Locations of the model parameter calibration points

\section{RESULTS AND DISCUSSION}

\section{Calibration in storm}

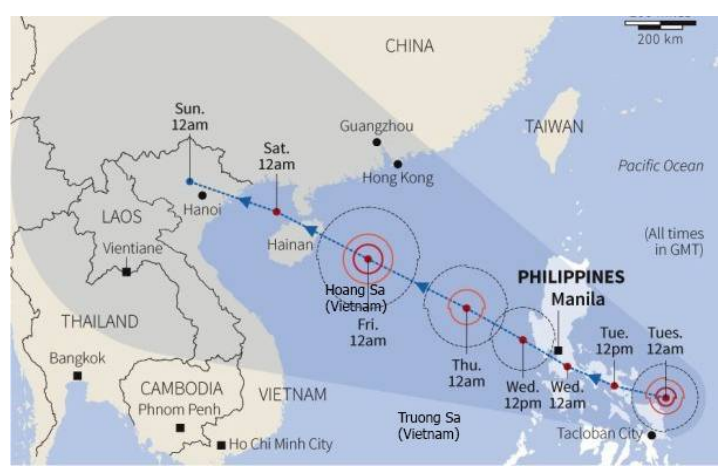

Fig. 2. The path of Rammasun Storm in Eastern Vietnam Sea

Calibration is performed with Rammasun Storm, this storm began on 12/7/2014 and finished on 19/7/2014, but it directly affected Eastern Vietnam Sea from 16-19/7/2014, so time period to simulate is from 0:00 on $15 / 7 / 2014$ to $23: 00$ on $21 / 7 / 2014$ (7 days). The total number of satellite data at the locations and time steps of simulation is 339 , the total number of data at MSP1 is 168 .

\section{Calibration with each parameter}

Calibration is performed by changing value of each parameter and keeping fixed default value of three other parameters (table 1). For each set of parameter values during parameter variation, the $G o F$ (eq. 3) provides a measure indicating how close the SWAN model results for that set are to observational data:

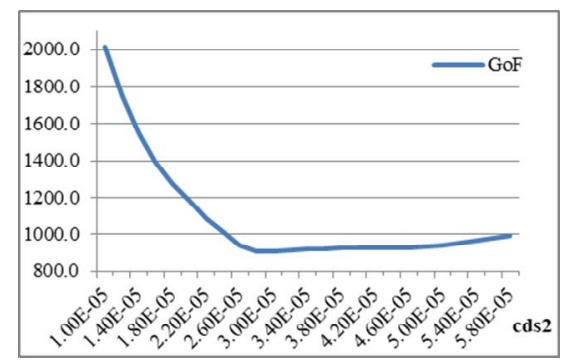

Fig. 3. Variation of the cost function $G o F$ with CDS2 (coefficient for determining the rate of whitecapping dissipation)

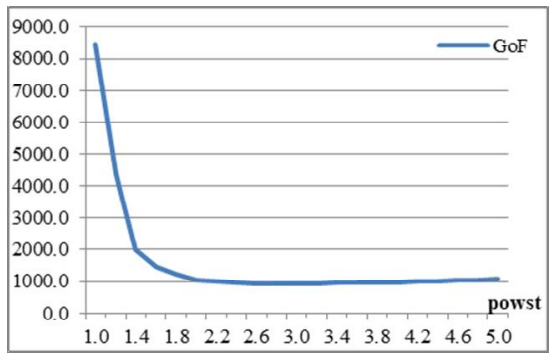

Fig. 4. Variation of the cost function $G o F$ with powst (power of steepness normalized with the wave steepness of a Pierson-Moskowitz spectrum)

The results show that changing the parameter powk (power of wave number normalized with the mean wave number) and the parameter cutfr (the ratio of this maximum frequency to the mean frequency) has little effect on the results. As a result, the $G o F$ value stays the same as 1060 with the parameter powk, the $G o F$ value changes very little when changing the parameter cutfr, from 1060.05 to 1060.6. Therefore, these two parameters will not be calibrated in the next step because these 


\section{Application of automated calibration method...}

parameters have negligible effect on the calculation results.

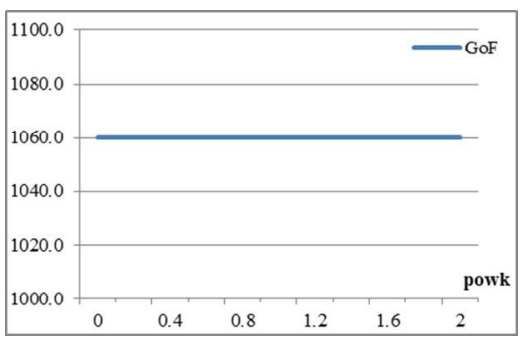

Fig. 5. Variation of the cost function GoF with powk (power of wave number normalized with the mean wave number)

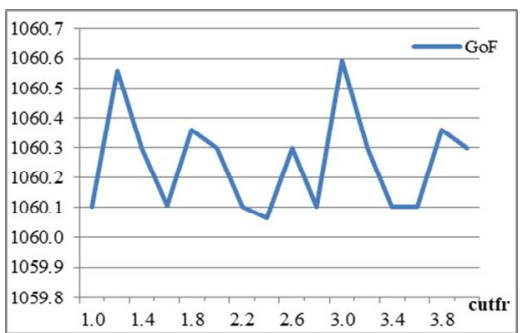

Fig. 6. Variation of the cost function $G o F$ with cutfr (the ratio of this maximum frequency to the mean frequency)
The GoF function varies greatly by changing the parameter CDS2 (coefficient for determining the rate of whitecapping dissipation) and the parameter powst (power of steepness normalized with the wave steepness of a Pierson-Moskowitz spectrum). The results also show that with values of CDS2 less than $2.0 \times 10^{-5}$ and powst less than 2 then the value of $G o F$ is very large, which is the large difference between simulation values and observation values. The optimal values in this case of CDS2 and powst are $2.8 \times 10^{-5}$ and 2.8. However, it cannot be asserted that this is the optimal set of parameters, so further calibrations are made to the two CDS2 and powst parameters simultaneously.

\section{Calibration of CDS2 and powst}

The calibration process is performed by modifying the two parameters CDS2 (from 2.0 $\times 10^{-5}$ to $4.8 \times 10^{-5}$, value step $2.0 \times 10^{-6}$ ) and powst (from 2.0 to 4.0 , value step 0.2 ). The table below shows the results of the 10 best options (the lowest $G o F$ and STD values) and the 10 worst options (the highest GoF and STD values) among the 240 calculation options.

Table 3. GoF values from low to high correspond to sets of parameters CDS2 and powst in Rammasun Storm

\begin{tabular}{|c|c|c|c|c|c|c|}
\hline No & Option & GoF & B & STD & powst & CDS2 \\
\hline 1 & 74 & 901 & -0.018 & 0.267 & 2.8 & $2.80 \mathrm{E}-05$ \\
\hline 2 & 90 & 903 & -0.028 & 0.267 & 2.8 & $3.00 \mathrm{E}-05$ \\
\hline 3 & 89 & 908 & -0.030 & 0.268 & 2.6 & $3.00 \mathrm{E}-05$ \\
\hline 4 & 88 & 909 & -0.034 & 0.269 & 2.4 & $3.00 \mathrm{E}-05$ \\
\hline 5 & 73 & 910 & -0.036 & 0.275 & 2.6 & 2.80E-05 \\
\hline 6 & 75 & 910 & -0.038 & 0.293 & 3.0 & $2.80 \mathrm{E}-05$ \\
\hline 7 & 91 & 913 & -0.042 & 0.295 & 3.0 & $3.00 \mathrm{E}-05$ \\
\hline 8 & 72 & 915 & -0.043 & 0.296 & 2.4 & $2.80 \mathrm{E}-05$ \\
\hline 9 & 87 & 920 & -0.051 & 0.301 & 2.2 & $3.00 \mathrm{E}-05$ \\
\hline 10 & 104 & 922 & -0.054 & 0.315 & 2.4 & 3.20E-05 \\
\hline 231 & 2 & 5788 & 0.302 & 0.995 & 1.2 & $2.00 \mathrm{E}-05$ \\
\hline 232 & 129 & 5981 & 0.208 & 1.046 & 1.0 & 3.60E-05 \\
\hline 233 & 113 & 5986 & 0.223 & 1.052 & 1.0 & $3.40 \mathrm{E}-05$ \\
\hline 234 & 97 & 6295 & 0.242 & 1.081 & 1.0 & 3.20E-05 \\
\hline 235 & 81 & 6804 & 0.264 & 1.117 & 1.0 & $3.00 \mathrm{E}-05$ \\
\hline 236 & 65 & 6915 & 0.283 & 1.139 & 1.0 & $2.80 \mathrm{E}-05$ \\
\hline 237 & 33 & 7392 & 0.293 & 1.159 & 1.0 & $2.40 \mathrm{E}-05$ \\
\hline 238 & 49 & 7392 & 0.307 & 1.179 & 1.0 & $2.60 \mathrm{E}-05$ \\
\hline 239 & 17 & 9240 & 0.364 & 1.287 & 1.0 & $2.20 \mathrm{E}-05$ \\
\hline 240 & 1 & 10085 & 0.393 & 1.336 & 1.0 & $2.00 \mathrm{E}-05$ \\
\hline
\end{tabular}




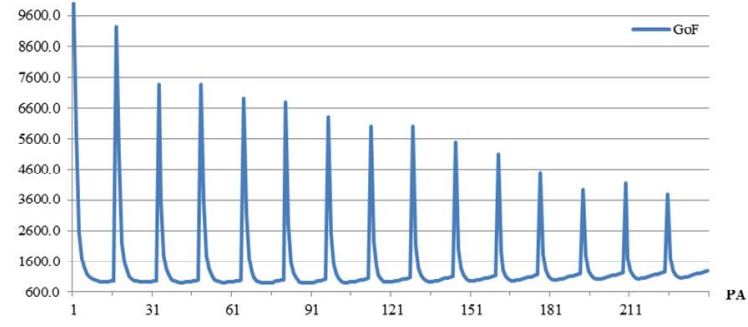

Fig. 7. Variation of the $G o F$ values with different sets of parameters CDS2 and powst in Rammasun Storm

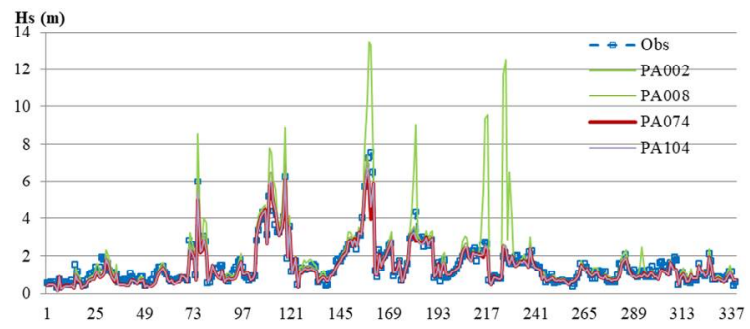

Fig. 8. Comparison of computed and satellite measured wave height in Rammasun Storm

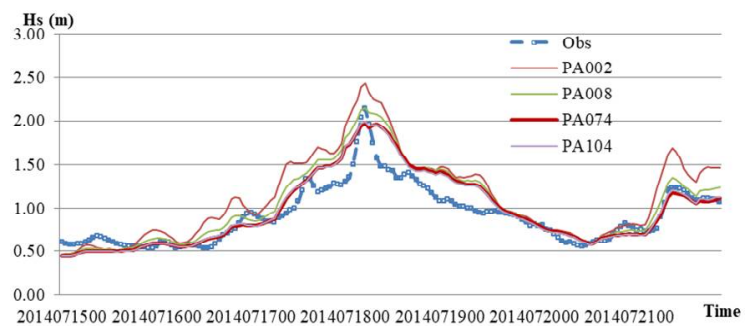

Fig. 9. Comparison of computed and MSP1 measured wave height in Rammasun Storm
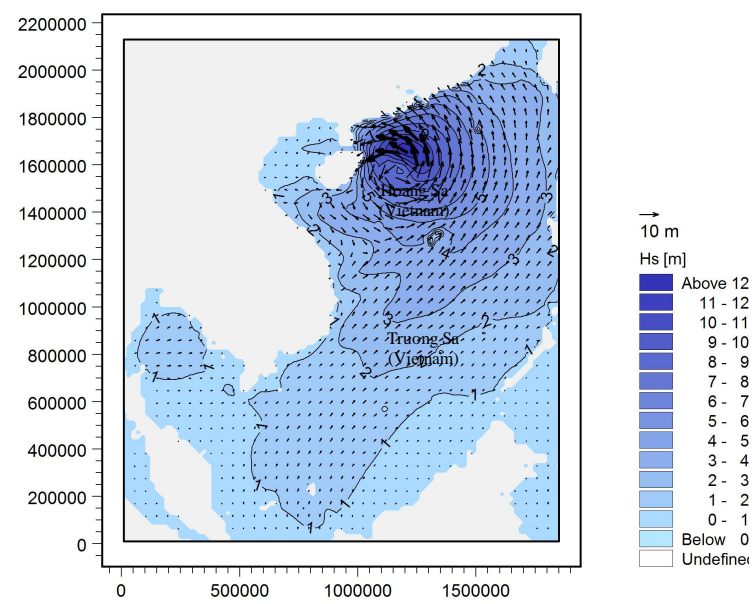

Fig. 10. Wave height and direction with $\mathrm{CDS} 2=2.36 \times 10^{-5}$ and powst $=2.0$ at 0.00 on $18 / 7 / 2014$ in Rammasun Storm

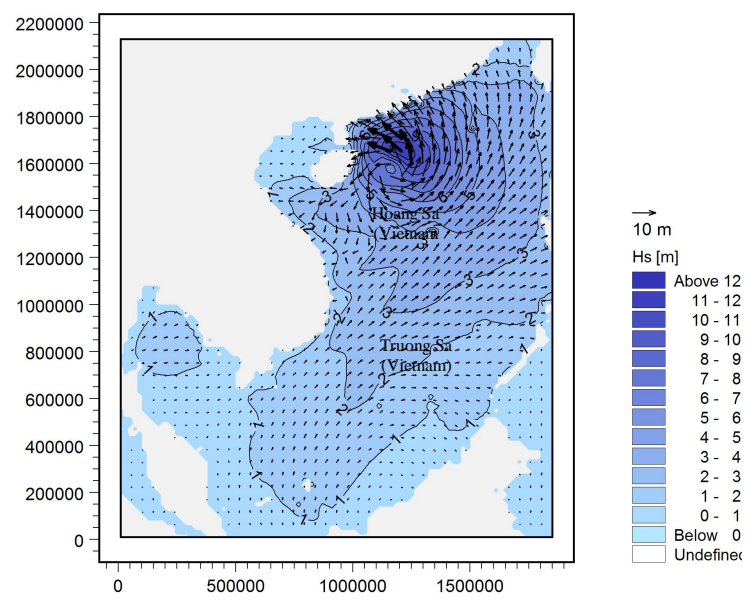

Fig. 11. Wave height and direction with CDS2 $=2.8 \times 10^{-5}$ and powst $=2.8$ at 0.00 on $18 / 7 / 2014$ in Rammasun Storm

The optimal values of the parameters CDS2 and powst are $2.8 \times 10^{-5}$ and 2.8 respectively and the optimal option is $74^{\text {th }}$. However, in the 10 best options in table 3, the values of the functions $G o F$ and STD are not much different so it is possible to use these parameter sets without much effect on calculation results, see fig. 8,9 .

\section{Calibration in monsoon}

In this case, the calibration is also made by changing the two parameters CDS2 (from $2.0 \times$ $10^{-5}$ to $4.8 \times 10^{-5}$, value step $2.0 \times 10^{-6}$ ) and powst (from 2.0 to 3.6, value step 0.2) with a separate sequence of independent data in monsoon. Calibration period is 7 days from 0:00 on $15 / 11 / 2014$ to $23: 00$ on $21 / 11 / 2014$. The total number of satellite data at the locations and time steps of simulation is 354 , the total number of data at MSP1 is 168 .

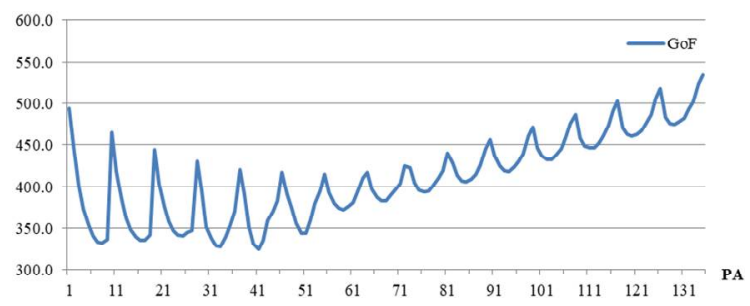

Fig. 12. Variation of the GoF value by CDS2 and powst parameters in monsoon 


\section{Application of automated calibration method...}

The table below shows the results of the 10 best options (the lowest GoF and STD values) and the 10 worst options (the highest GoF and STD values) out of 135 simulations.

Table 4. GoF values from low to high correspond to CDS2 and powst parameters in monsoon

\begin{tabular}{|c|c|c|c|c|c|c|}
\hline No & Option & GoF & B & STD & powst & CDS2 \\
\hline 1 & 41 & 325 & -0.061 & 0.136 & 2.8 & 2.80E-05 \\
\hline 2 & 33 & 328 & -0.063 & 0.144 & 3.0 & $2.60 \mathrm{E}-05$ \\
\hline 3 & 32 & 330 & -0.092 & 0.150 & 2.8 & $2.60 \mathrm{E}-05$ \\
\hline 4 & 40 & 331 & -0.096 & 0.164 & 2.6 & $2.80 \mathrm{E}-05$ \\
\hline 5 & 8 & 332 & -0.098 & 0.177 & 3.4 & $2.00 \mathrm{E}-05$ \\
\hline 6 & 7 & 334 & -0.107 & 0.183 & 3.2 & $2.00 \mathrm{E}-05$ \\
\hline 7 & 42 & 336 & -0.116 & 0.190 & 3.0 & $2.80 \mathrm{E}-05$ \\
\hline 8 & 17 & 336 & -0.124 & 0.192 & 3.4 & $2.20 \mathrm{E}-05$ \\
\hline 9 & 16 & 336 & -0.126 & 0.197 & 3.2 & $2.20 \mathrm{E}-05$ \\
\hline 10 & 9 & 337 & -0.128 & 0.199 & 3.6 & $2.00 \mathrm{E}-05$ \\
\hline 126 & 108 & 487 & -0.208 & 0.172 & 3.6 & 4.20E-05 \\
\hline 127 & 116 & 491 & -0.210 & 0.171 & 3.4 & 4.40E-05 \\
\hline 128 & 132 & 493 & -0.215 & 0.169 & 3.0 & 4.80E-05 \\
\hline 129 & 1 & 494 & 0.012 & 0.269 & 2.0 & $2.00 \mathrm{E}-05$ \\
\hline 130 & 133 & 501 & -0.219 & 0.171 & 3.2 & 4.80E-05 \\
\hline 131 & 117 & 503 & -0.216 & 0.173 & 3.6 & 4.40E-05 \\
\hline 132 & 125 & 506 & -0.218 & 0.172 & 3.4 & 4.60E-05 \\
\hline 133 & 126 & 518 & -0.223 & 0.174 & 3.6 & 4.60E-05 \\
\hline 134 & 134 & 523 & -0.225 & 0.173 & 3.4 & 4.80E-05 \\
\hline 135 & 135 & 535 & -0.230 & 0.175 & 3.6 & 4.80E-05 \\
\hline
\end{tabular}

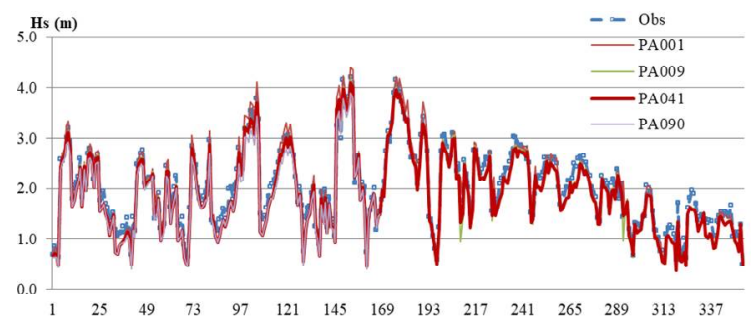

Fig. 13. Comparison of computed and satellite measured wave height in monsoon

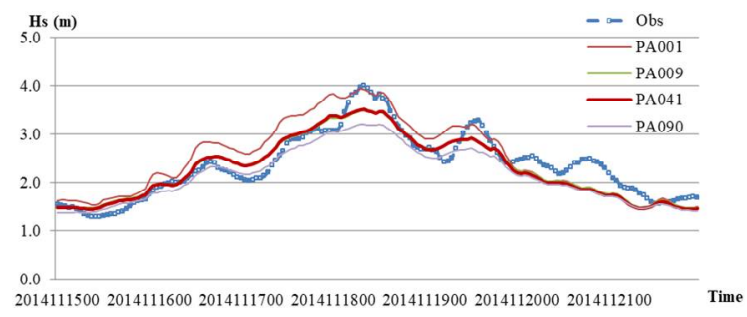

Fig. 14. Comparison of computed and MSP1 measured wave height in monsoon

As with previous simulations, the optimal values of the parameters CDS2 and powst are $2.8 \times 10^{-5}$ and 2.8 , respectively and the optimal option is option 41 . However in the 10 optimal options in table 4, the values of $G o F$ function are not much different so it is possible to use these sets of parameters without affecting much calculation results.

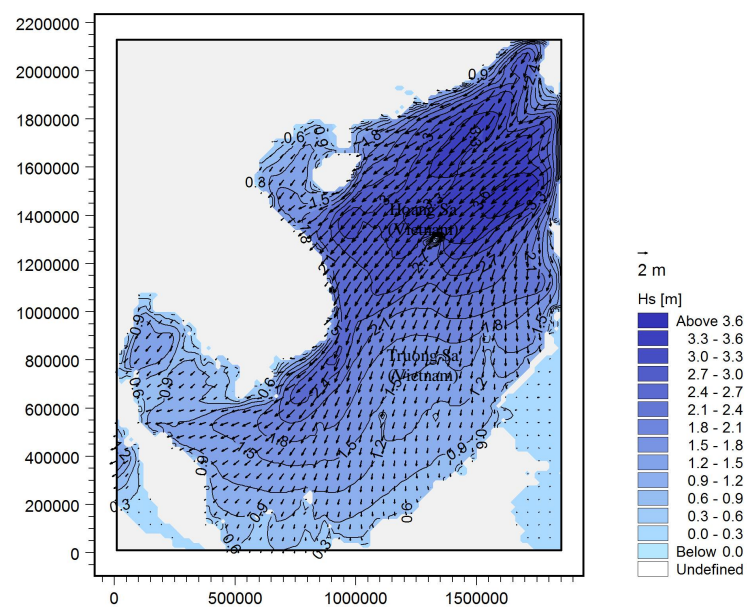

Figure 15. Wave height and direction with CDS2 $=2.36 \times 10^{-5}$ and powst $=2.0$ at 0.00 on 19/11/2014 in monsoon 


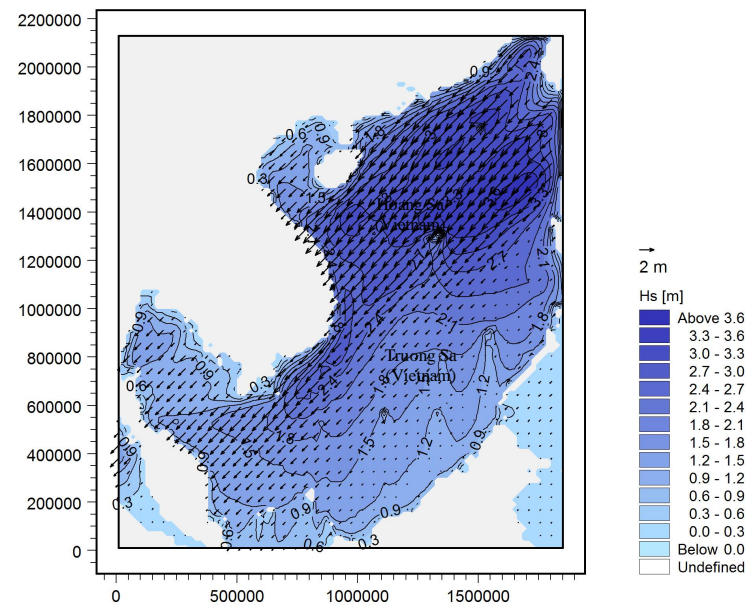

Fig. 16. Wave height and direction with CDS2 $=2.8 \times 10^{-5}$ and powst $=2.8$ at 0.00 on $19 / 11 / 2014$ in monsoon

\section{CONCLUSIONS}

Automated calibration method SWAN-CI of OpenDA is useful tool that can be used to calibrate many of the SWAN model's parameters with minimization of the distance between the observation and the model prediction by the cost function $G o F$.

In this study, model parameters were calibrated with two data series corresponding to Rammasun Storm in July 2014 and monsoon in November 2014. The total independent and combined simulations are 300 and 135 options, respectively. And the total calibrated locations are 175 points.

The simulated results show that the wave field simulated by SWAN in storm and monsoon is quite good. The parameter powk (power of wave number normalized with the mean wave number) and the parameter cutfr (the ratio of this maximum frequency to the mean frequency) have little effects on the results of the simulations, while the parameter CDS2 (coefficient for determining the rate of whitecapping dissipation) and the parameter powst (power of steepness normalized with the wave steepness of a Pierson-Moskowitz spectrum) have strong effects on the results. Although the optimal parameter sets with CDS2 and powst are $2.8 \times 10^{-5}$ and 2.8, respectively, some other optimal parameter sets as shown in tables 3, 4 can be used to forecast.

\section{REFERENCES}

1. Duong Cong Dien, Nguyen Manh Hung, 2006. Calibration and verification of a storm wave model in the coastal zones of the Eastern Vietnam Sea. Congress of the Vietnam Association for Fluid Mechanics.

2. Hung, N. M., Thuy, D. L., and Dien, D. C., 2005. Storm wave modeling with swan comparison of measurement data and modeling results for the storm muifa 11/2004. Vietnam Journal of Mechanics, 27(4), 229-239.

3. Booij, N., Ris, R. C., and Holthuijsen, L. H., 1999. A third-generation wave model for coastal regions: 1. Model description and validation. Journal of geophysical research: Oceans, 104(C4), 7649-7666.

4. Komen, G. J., Cavaleri, L., Donelan, M., Hasselmann, K., Hasselmann, S., and Janssen, P. A. E. M., 1994. Dynamics and modelling of ocean waves. Cambridge Univ. Press, Cambridge, U. K.

5. Phillips, O. M., 1957. On the generation of waves by turbulent wind. Journal of Fluid Mechanics, 2(5), 417-445.

6. Miles, J. W., 1957. On the generation of surface waves by shear flows. Journal of Fluid Mechanics, 3(2), 185-204.

7. Verlaan, M., 2016. Open DA User Documentation. TU Delft.

8. Wenneker, I., Dhondia, J., Hummel, S., Verlaan, M., Gerritsen, H., 2008. User Manual Calibration Instrument SWAN. Delft Hydraulics.

9. http://ngdc.noaa.gov.

10. http://polar.ncep.noaa.gov.

11. http://odes.altimetry.cnes.fr. 